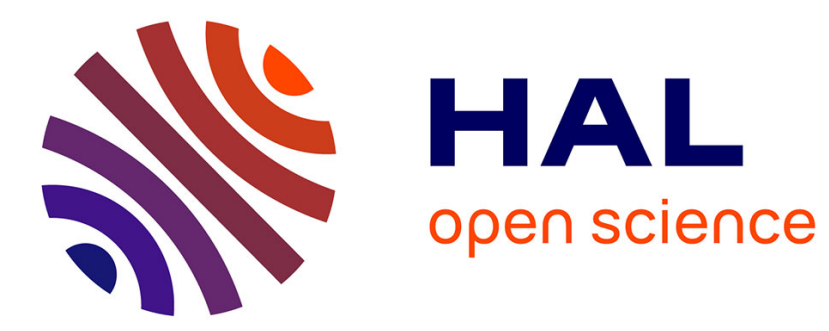

\title{
A new experimental setup to study the structure of curiosity-driven exploration in humans
}

Brice Miard, Pierre Rouanet, Jonathan Grizou, Manuel Lopes, Jacqueline Gottlieb, Adrien Baranes, Pierre-Yves Oudeyer

\section{- To cite this version:}

Brice Miard, Pierre Rouanet, Jonathan Grizou, Manuel Lopes, Jacqueline Gottlieb, et al.. A new experimental setup to study the structure of curiosity-driven exploration in humans. ICDL-EPIROB 2014, Oct 2014, Genoa, Italy. hal-01061682

HAL Id: hal-01061682

https://hal.inria.fr/hal-01061682

Submitted on 8 Sep 2014

HAL is a multi-disciplinary open access archive for the deposit and dissemination of scientific research documents, whether they are published or not. The documents may come from teaching and research institutions in France or abroad, or from public or private research centers.
L'archive ouverte pluridisciplinaire HAL, est destinée au dépôt et à la diffusion de documents scientifiques de niveau recherche, publiés ou non, émanant des établissements d'enseignement et de recherche français ou étrangers, des laboratoires publics ou privés. 


\title{
A new experimental setup to study the structure of curiosity-driven exploration in humans
}

\author{
Brice Miard, Pierre Rouanet, Jonathan Grizou, \\ Manuel Lopes, Jacqueline Gottlieb, Adrien Baranes, Pierre-Yves Oudeyer \\ Flowers Team: Inria, Ensta Paris-Tech - Department of Neuroscience, Columbia University \\ firstname.lastname@inria.fr
}

\section{INTRODUCTION}

Curiosity is a key element of human development, driving us to explore spontaneously novel objects, activities and environments [1]. Curiosity-driven exploration strategies permit us to interact, learn and evolve quickly in an open ended world. It is thus an important challenge to understand the fundamental mechanisms of spontaneous exploration and curiosity in humans.

One of the first experiments on this topic was made by Harlow, where monkeys played with simple 2D puzzles to highlight the relation between complexity, motivation and learning [2]. Another early example is McReynolds et al. who created the "curiosity box" where identical boxes, with different toys inside, are presented to young children [3]. Recently, an experiment was made with infants to study action selection guided by intrinsic motivations with a mechatronic board. In this study, subjects have to learn the relation between actions on pushbuttons and the opening of boxes [4]. They showed significant differences in exploration strategy (sensorimotor vs a more learning directed exploration) between three and four years old infants. Despite a number of other psychology and neuroscience experiments in humans and monkeys, we still know little about the precise mechanisms of curiosity [1].

Many computational models of curiosity have been elaborated [1], [5]. Some of these models specifically targeted the modeling of curiosity and its role in human sensorimotor and cognitive development, showing how it can generate automatically behavioral and cognitive developmental structures sharing interesting similarities with infant development [6], [7]. These lines of work allowed to identify the wide diversity of potential mechanisms that could be at play to drive spontaneous exploration [8]. We focus here on the intrinsic motivation mechanisms driving exploration, i.e. the processes allowing an agent to choose itself goals when freely involved in a task.

Novel hypotheseses have been formulated such that curiosity-driven sensorimotor exploration could be organized as to maximize learning progress [9], [10], which is different from more classical hypothesis conceptualizing curiosity as a drive to maximize uncertainty of novelty. Yet, experimental setups designed so far in the literature do not allow to separate between these hypotheses. A major research challenge is thus to design experimental setups which could allow to confirm or invalidate individual hypotheses. Furthermore, it is important to note that intrinsic motivation mechanisms could be influenced when asked to behave according to a specific protocol. This will be discussed in details when describing our experimental setup.

Here, we make a step in this direction by presenting an exploratory study with humans designed to analyze and measure properties of curiosity-driven exploration of a priori unknown sensorimotor spaces. More specifically, we are interested in the relation between exploration and learning progress.

\section{EXPERIMENTAL SETUP}
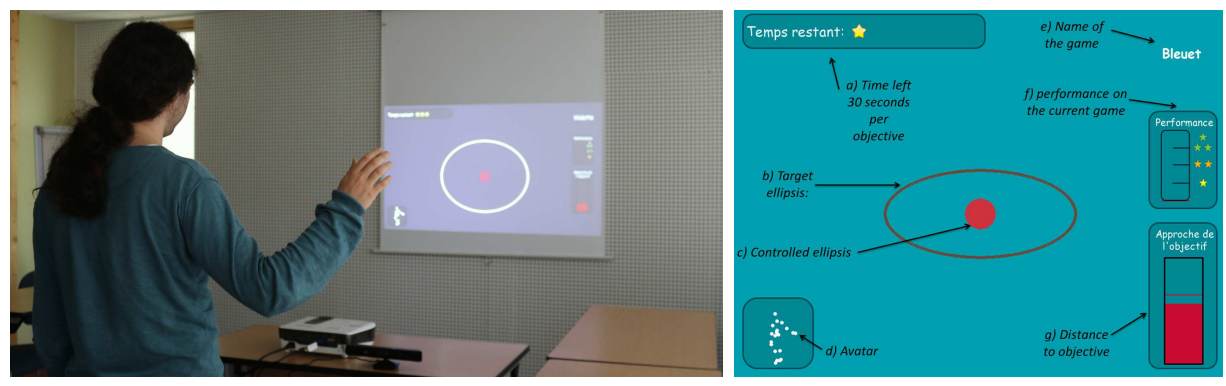

Fig. 1. a) Subjects are exploring how to control an ellipsis displayed on the screen in front of them by moving their body joints tracked by a Kinect device. b) Details of the interface shown to the user on a screen in front of them: in particular, you can see the controlled ellipsis (in red) and the target one (in brown).

This experimental setup is designed as a game setting human subjects into an intrinsically motivated activity [11]. Participants can freely explore and shift between several games, each being about finding a mapping between their movements (body joints 
tracked by a kinect) and a 2D shape displayed on a screen (an ellipsis). We will first describe the setup, then present the ongoing users study and preliminary results. In each game, they have to match the shape they control with randomly generated goals (see figure 1b). When reached, i.e. when the controlled shape is maintained close enough of the target for one second, a new goal is automatically generated. Objectives change every 30 seconds when not reached. Additional performance indicators were added on the screen interface in order to improve the subject's, implication and comprehension (see figure 1b) [11].

Each of the four games, while visually similar, differs by the difficulty of the underlying mapping (more or less easy to learn). Typically, for each of these mappings, the axes of the ellipsis are controlled by one of the body's joint: e.g. the major axis will be controlled by the pitch angle of the left shoulder while the minor axis will be controlled by the yaw angle of the right elbow. Note that in one of the game, a single joint is used to control both axis making the ellipsis a circle. Users are entirely free to change from one game to another one, thanks to a tablet, when and how often they want. It is important to note here that while they are extrinsically motivated to play each game (protocol, score...), they are entirely free to change games whenever they want. Thus, as we focus on the transition and how they divide their time among those four games, we assume that we are still measuring intrinsically motivated behaviors.

The following protocol was used for the ongoing user's study, so far about twenty participants played our game. First, participants had to fill in a questionnaire (on a five Likert scale) aiming at highlighting personality and skill profiles (e.g. practice of body expression activities such as dance or theatre). This pre-questionnaire also include the CEI2 questionnaire [12], a psychological test designed to evaluate level of curiosity. Then, they could play the four different games for a total of 15 minutes. Afterwards, they had to do a quick test, play each game for one minute and reach the highest score as possible. Finally, they had to fill in another questionnaire to collect their subjective point of view of their user's experience (difficulty, time spent on each games, progress, expected progress).

During all the experiments, we also record each time they reach a goal and each time they change games. We can thus compute their learning progression by summing the number of objectives succeeded in a time window. We also recorded the movement of their 20 articulations of their bodies in 3D at $10 \mathrm{~Hz}$ allowing the study of exploration strategy such as the freezing/unfreezing of degrees of freedom. A video presenting the setup and the experiments is available here: https://vimeo.com/95383753.

\section{FUTURE WORK}

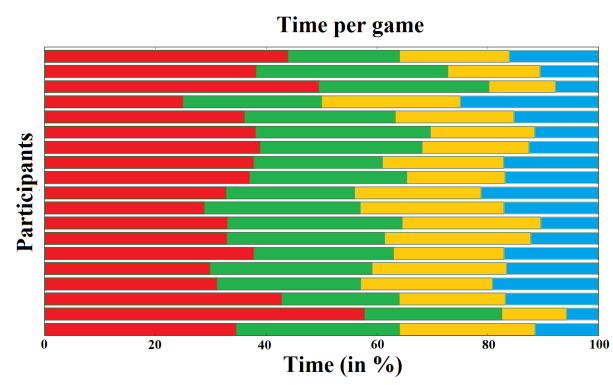

Fig. 2. Percentage of time spent by subjects on the 4 differents games.

We analyzed data from 20 participants selected among staffs and students at Inria. We observe a different repartition of the time spent per game. Our future work will consist of trying to infer the reasons from the when and why the participants change game from the data. We particularly want to investigate whether or not the hyphothesis that activity change is related to learning progress.

\section{ACKNOWLEDGEMENT}

We would like to thanks Julie Golliot for helping us designing the experimental setup and protocol. This research was partially funded by Inria Neurocuriosity and ERC 2400007.

\section{REFERENCES}

[1] J. Gottlieb, P.-Y. Oudeyer, M. Lopes, and A. Baranes, "Information-seeking, curiosity, and attention: computational and neural mechanisms," Trends in cognitive sciences, vol. 17, no. 11, pp. 585-593, 2013.

[2] H. F. Harlow, "Learning and satiation of response in intrinsically motivated complex puzzle performance by monkeys." Journal of Comparative and Physiological Psychology, vol. 43, no. 4, p. 289, 1950.

[3] P. McReynolds, M. Acker, and C. Pietila, "Relation of object curiosity to psychological adjustment in children," Child Development, pp. 393-400, 1961.

[4] F. Taffoni, E. Tamilia, V. Focaroli, D. Formica, L. Ricci, G. Di Pino, G. Baldassarre, M. Mirolli, E. Guglielmelli, and F. Keller, "Development of goal-directed action selection guided by intrinsic motivations: an experiment with children," Experimental brain research, pp. 1-11, 2014.

[5] G. Baldassarre and M. Mirolli, Intrinsically motivated learning in natural and artificial systems. Springer, 2013.

[6] C. Moulin-Frier, S. M. Nguyen, and P.-Y. Oudeyer, "Self-Organization of Early Vocal Development in Infants and Machines: The Role of Intrinsic Motivation," Frontiers in Psychology, vol. 4, no. 1006, 2013. [Online]. Available: http://hal.inria.fr/hal-00927940

[7] P.-Y. Oudeyer and L. Smith, "How evolution may work through curiosity-driven developmental process," Topics in Cognitive Science, 2014.

[8] P.-Y. Oudeyer and F. Kaplan, "What is intrinsic motivation? a typology of computational approaches," Frontiers in neurorobotics, vol. 1, 2007.

[9] J. Schmidhuber, "Curious model-building control systems," in Neural Networks, 1991. 1991 IEEE International Joint Conference on. IEEE, 1991.

[10] P.-Y. Oudeyer, F. Kaplan, and V. V. Hafner, "Intrinsic motivation systems for autonomous mental development," Evolutionary Computation, IEEE Transactions on, vol. 11, no. 2, pp. 265-286, 2007.

[11] T. W. Malone and M. R. Lepper, "Making learning fun: A taxonomy of intrinsic motivations for learning," Aptitude, learning, and instruction, 1987.

[12] T. B. Kashdan, M. W. Gallagher, P. J. Silvia, B. P. Winterstein, W. E. Breen, D. Terhar, and M. F. Steger, "The curiosity and exploration inventory-ii: Development, factor structure, and psychometrics," Journal of research in personality, vol. 43, no. 6, pp. 987-998, 2009. 